\title{
Modulated dust-acoustic wave packets in an opposite polarity dusty plasma system
}

\author{
S. Jahan ${ }^{a}$, N. A. Chowdhury, A. Mannan, and A. A. Mamun \\ Department of Physics, Jahangirnagar University, \\ Savar, Dhaka-1342, Bangladesh. \\ *Email: jahan88phy@gmail.com ${ }^{a}$
}

\begin{abstract}
The nonlinear propagation of the dust-acoustic bright and dark envelope solitons in an opposite polarity dusty plasma system (composed of non-extensive $q$-distributed electrons, isothermal ions, and positively as well as negatively charged warm dust) have been theoretically investigated. The reductive perturbation method (which is valid for a small, but finite amplitude limit) is employed to derive the nonlinear Schrödinger equation. Two types of modes (namely, fast and slow dustacoustic (DA) modes) have been observed. The conditions for the modulational instability (MI) and its growth rate in the unstable regime of the DA waves are significantly modified by the effects of non-extensive electrons, dust mass, temperatures of different plasma species, etc. The implications of the obtained results from our current investigation in space (viz. Jupiters magnetosphere, upper mesosphere, and comets tails) and laboratory (e.g. direct current and radio-frequency discharges, plasma processing reactors, fusion plasma devices, and solid-fuel combustion products, etc.) dusty plasmas are briefly discussed.
\end{abstract}

\section{LEAD PARAGRAPH}

The nonlinear propagation of the dust-acoustic bright and dark envelope solitons in an opposite polarity dusty plasma system (containing isothermal ions, non-extensive $q$-distributed electrons, and positively as well as negatively charged warm dust) has been studied to identify some new features (e.g. conditions for the modulational instabiliuty of the dust-acoustic waves (DAWs), growth rate, formation of the bright and dark envelope solitons, etc.) of the DAWs. These new features should be useful for understanding the nonlinear electrostatic disturbances in space and laboratory dusty plasmas.

\section{INTRODUCTION}

Now-a-days, a great deal of interest has been devoted to the new and fascinating field of dusty plasma due to their existence in space 1-4 and massive applications in laboratory plasmas [5 7]. The dusty plasma is generally considered to be an ensemble of dust particles, free electrons, and ions, which has been found widely in planetary rings, asteroid zones [6], cometary tails, magnetosphere as well as the lower part of the earth's atmosphere [3, 4]. However, the co-existence of opposite polarity dusty in plasmas, introduces a new dusty plasma model called "opposite polarity dusty plasma" (OPDP) [8], whose main species are positively and negatively charged warm massive dust [8]. The exclusive property of this OPDP, which makes it completely unique from other plasmas (viz. electron ion and electron-positron plasmas) is that the ratio of the size of positively charged dust to that of negatively charged dust can be smaller [9] or larger [10] or equal to unity [10]. Dust species can be charged either positively or negatively, because of different charging processes, like photo-ionization, secondary electron emission, thermionic emission, and collection of plasma particles (electrons and ions), etc. The existence of positively charged dust has been observed in different regions of space (e.g. Jupiter's magnetosphere [11], cometary tails [9, 10, 12, 13], upper mesosphere 14], etc). There are three main processes by which dust grains become positively charged, namely, [15, 16] (a) secondary emission of electrons from the surface of the dust grains; (b) thermionic emission induced by the radiative heating; and (c) photoemission in the presence of a flux of ultraviolet photons. There is also direct evidence for the co-existence of both positively and negatively charged dust particles in different regions of space plasmas (viz. cometary tails [12, 13], upper mesosphere [14], Jupiter's magnetosphere [11, 13, 16, 17], etc.) and laboratory devices (e.g. direct current and radio-frequency discharges, plasma processing reactors, fusion plasma devices, solidfuel combustion products, etc [1, 18]). In space and astrophysical sectors, if the plasma particles move very fast compared to their thermal velocities [19] then the Maxwellian distribution is no longer valid. For that reason, Tsallis proposed the non-extensive statistics 20, 21], which is the generalisation of Boltzmann-Gibbs-Shannon entropy. The importance of Tsallis statistics lies with describing the system of long range interactions, such as, plasmas and dusty plasmas [21].

The researchers are focused on wave dynamics, specifically, dust-acoustic (DA) waves (DAWs), dust-acoustic rogue waves (DARWs), and dust ion-acoustic waves (DIAWs) in understanding electrostatic density perturbations and potential structures (viz. soliton, shock, vortices, and rogue waves [17, 22 24]). The DAWs [6] have low phase velocity (where the inertia is provided by the dust mass and restoring force is provided by the thermal pressure of electrons and ions [17]) in comparison with the electron and ion thermal velocities. The research on modulational instability (MI) of DAWs in nonlinear and dispersive mediums have been increasing significantly due to their existence in astrophysics, space 
physics 25] as well as in application in many laboratory situations [26]. A great number of researchers have used the nonlinear Schrödinger (NLS) equation, which governs the dynamics of the DAWs and the formation of the envelope solitons [26]. Sayed and Mamun [27] studied the finite solitary potential structures that exist in OPDP. ElTaibany [28] examined the DAWs in inhomogeneous fourcomponent dusty plasmas with opposite charge polarity dust grains and observed that only compressive soliton is created corresponding to fast DAWs velocities. To the best knowledge of the authors, no attempt has been made on MI, and corresponding dark and bright envelope solitons associated with the DAWs containing non-extensive $q$-distributed electrons, isothermal ions, and positively and negatively charged warm massive dust. Therefore, in our article, we will derive the NLS equation by employing the reductive perturbation method and examine the conditions for the MI of the DAWs (in which inertia is provided by the dust mass and restoring force is provided from the thermal pressure of non-extensive $q$-distributed electrons and isothermal ions).

The manuscript is organized as the following fashion: The governing equations of our considered plasma model are stated in Sec. III. The NLS equation is derived in Sec. IV] The stability of DAWs and envelope solitons are examined in Sec. V andVI, respectively. Finally, the summary of our discussion is provided in Sec. VII]

\section{MODEL EQUATIONS}

In this paper, we consider a collisionless, fully ionized, unmagnetized four component dusty plasma system formed of non-extensive q-distributed electrons (charge $-e$, mass $m_{e}$ ), isothermal ions (charge $+e$; mass $m_{i}$ ) and inertial warm negatively charged dust particles (charge $q_{1}=-z_{1} e$, mass $\left.m_{1}\right)$ as well as positively charged warm dust particles (charge $q_{2}=+z_{2} e$; mass $m_{2}$ ); where $z_{1}$ $\left(z_{2}\right)$ is the charge state of the negatively (positively) charged warm dust particles. The negatively and positively charged warm dust particles can be displayed by continuity and momentum equations, respectively, as given below:

$$
\begin{aligned}
& \frac{\partial n_{1}}{\partial t}+\frac{\partial}{\partial x}\left(n_{1} u_{1}\right)=0, \\
& \frac{\partial u_{1}}{\partial t}+u_{1} \frac{\partial u_{1}}{\partial x}=\frac{z_{1} e}{m_{1}} \frac{\partial \varphi}{\partial x}-\frac{1}{m_{1} n_{1}} \frac{\partial p_{1}}{\partial x}, \\
& \frac{\partial n_{2}}{\partial t}+\frac{\partial}{\partial x}\left(n_{2} u_{2}\right)=0, \\
& \frac{\partial u_{2}}{\partial t}+u_{2} \frac{\partial u_{2}}{\partial x}=-\frac{z_{2} e}{m_{2}} \frac{\partial \varphi}{\partial x}-\frac{1}{m_{2} n_{2}} \frac{\partial p_{2}}{\partial x},
\end{aligned}
$$

where $n_{1}\left(n_{2}\right)$ is the number densities of the negatively (positively) charged warm dust particles; $t(x)$ is the time (space) variable; $u_{1}\left(u_{2}\right)$ is the fluid speed of the negatively (positively) charged warm dust species; $e$ is the magnitude of the charge of the electron; $\varphi$ is the electro- static wave potential; and $p_{1}\left(p_{2}\right)$ is the adiabatic pressure of the negatively (positively) charged warm dust particles. The system is closed through Poisson's equation, stated as

$$
\frac{\partial^{2} \varphi}{\partial x^{2}}=4 \pi e\left(n_{e}-n_{i}+z_{1} n_{1}-z_{2} n_{2}\right),
$$

where $n_{i}$ and $n_{e}$ are, respectively, the ion and electron number densities. The quasi-neutrality condition at equilibrium can be shown as

$$
n_{i 0}+z_{2} n_{20}=n_{e 0}+z_{1} n_{10},
$$

where $n_{i 0}, n_{20}, n_{e 0}$, and $n_{10}$ are the equilibrium number densities of the isothermal ions, positively charged warm dust particles, non-extensive $q$-distributed electrons, and negatively charged warm dust particles, respectively. Now, in terms of normalized variables, namely, $N_{1}=$ $n_{1} / n_{10}, N_{2}=n_{2} / n_{20}, U_{1}=u_{1} / C_{d 1}$ (with $C_{d 1}$ being the sound speed of the negatively charged warm dust particles), $U_{2}=u_{2} / C_{d 1} ; \phi=e \varphi / T_{i}$ (with $T_{i}$ being the temperature of the isothermal ion); $T=t \omega_{p d 1}$ (with $\omega_{p d 1}$ being the plasma frequency of the negatively charged warm dust particles), $X=x / \lambda_{D d 1}$ (with $\lambda_{D d 1}$ being the Debye length of the negatively charged warm dust species); $C_{d 1}=\left(z_{1} T_{i} / m_{1}\right)^{1 / 2}, \omega_{p d 1}=\left(4 \pi e^{2} z_{1}^{2} n_{10} / m_{1}\right)^{1 / 2} ; \lambda_{D d 1}=$ $\left(T_{i} / 4 \pi e^{2} z_{1} n_{10}\right)^{1 / 2} ; p_{1}=p_{10}\left(n_{1} / n_{10}\right)^{\gamma}$ [with $p_{10}$ being the equilibrium adiabatic pressure of the negatively charged warm dust particles and $\gamma=(N+2) / N$, where $N$ is the degree of freedom, for one-dimensional case, $N=1$ so that $\gamma=3], p_{10}=n_{10} T_{1}$ (with $T_{1}$ being the temperature of the negatively charged warm dust particles); $p_{2}=p_{20}\left(n_{2} / n_{20}\right)^{3}$ (with $p_{20}$ being the equilibrium adiabatic pressure of the positively charged warm dust particles), and $p_{20}=n_{20} T_{2}$ (with $T_{2}$ being the temperature of the positively charged warm dust particles). After normalization, Eqs. (10) - (5) becomes

$$
\begin{aligned}
& \frac{\partial N_{1}}{\partial T}+\frac{\partial}{\partial X}\left(N_{1} U_{1}\right)=0, \\
& \frac{\partial U_{1}}{\partial T}+U_{1} \frac{\partial U_{1}}{\partial X}+3 \sigma_{1} N_{1} \frac{\partial N_{1}}{\partial X}=\frac{\partial \phi}{\partial X}, \\
& \frac{\partial N_{2}}{\partial T}+\frac{\partial}{\partial X}\left(N_{2} U_{2}\right)=0, \\
& \frac{\partial U_{2}}{\partial T}+U_{2} \frac{\partial U_{2}}{\partial X}+3 \sigma_{2} N_{2} \frac{\partial N_{2}}{\partial X}=-\alpha \frac{\partial \phi}{\partial X}, \\
& \frac{\partial^{2} \phi}{\partial X^{2}}=\left(\mu_{i}+\beta-1\right) N_{e}-\mu_{i} N_{i}+N_{1}-\beta N_{2},
\end{aligned}
$$

where $\sigma_{1}=T_{1} / z_{1} T_{i}, \quad \sigma_{2}=m_{1} T_{2} / z_{1} m_{2} T_{i}, \alpha=$ $m_{1} z_{2} / m_{2} z_{1}, \beta=z_{2} n_{20} / z_{1} n_{10}$, and $\mu_{i}=n_{i 0} / z_{1} n_{10}$. The number densities of the non-extensive q-distributed [17] electron can be given by the following normalized equation

$$
N_{e}=[1+(q-1) \delta \phi]^{\frac{q+1}{2(q-1)}},
$$

where $\delta=T_{i} / T_{e}$ and $q$ is the non-extensive parameter describing the degree of non-extensivity, i.e., $q=1$ indicates the Maxwellian distribution, whereas $q<1$ refers 
to the super-extensivity, and the opposite condition $q>1$ corresponds to the sub-extensivity [29]. The number densities of the iso-thermally distributed [17] ion can be represented as

$$
N_{i}=\exp (-\phi)
$$

Now, substituting Eqs. (12) and (13) into Eq. (11), and extending up to third order in $\phi$, we can write

$$
\frac{\partial^{2} \phi}{\partial X^{2}}+\beta N_{2}-N_{1}=\beta-1+\gamma_{1} \phi+\gamma_{2} \phi^{2}+\gamma_{3} \phi^{3}+. .
$$

where

$$
\begin{aligned}
& \gamma_{1}=\frac{\left(\beta+\mu_{i}-1\right)(q+1) \delta}{2}+\mu_{i}, \\
& \gamma_{2}=\frac{\left(\beta+\mu_{i}-1\right)(q+1)(3-q) \delta^{2}}{8}-\frac{\mu_{i}}{2}, \\
& \gamma_{3}=\frac{\left(\beta+\mu_{i}-1\right)(q+1)(q-3)(3 q-5) \delta^{3}}{48}+\frac{\mu_{i}}{6} .
\end{aligned}
$$

The left hand side of Eq. (14), is the contribution of electron and ion species.

\section{DERIVATION OF THE NLS EQUATION}

We will use the reductive perturbation method to derive the NLS equation to know the modulation of the DAWs. Let us consider, the stretched co-ordinate [17] as

$$
\begin{aligned}
& \xi=\epsilon\left(X-V_{g} T\right), \\
& \tau=\epsilon^{2} T,
\end{aligned}
$$

where $V_{g}$ is the envelope group velocity and $\epsilon(0<\epsilon<1)$ is a small (real) parameter. The dependent variables [17] can be written as

$$
\begin{aligned}
& N_{1}=1+\sum_{m=1}^{\infty} \epsilon^{(m)} \sum_{l=-\infty}^{\infty} N_{1 l}^{(m)}(\xi, \tau) \exp (i l \Upsilon), \\
& U_{1}=\sum_{m=1}^{\infty} \epsilon^{(m)} \sum_{l=-\infty}^{\infty} U_{1 l}^{(m)}(\xi, \tau) \exp (i l \Upsilon), \\
& N_{2}=1+\sum_{m=1}^{\infty} \epsilon^{(m)} \sum_{l=-\infty}^{\infty} N_{2 l}^{(m)}(\xi, \tau) \exp (i l \Upsilon), \\
& U_{2}=\sum_{m=1}^{\infty} \epsilon^{(m)} \sum_{l=-\infty}^{\infty} U_{2 l}^{(m)}(\xi, \tau) \exp (i l \Upsilon), \\
& \phi=\sum_{m=1}^{\infty} \epsilon^{(m)} \sum_{l=-\infty}^{\infty} \phi_{l}^{(m)}(\xi, \tau) \exp (i l \Upsilon),
\end{aligned}
$$

where $\Upsilon=k X-\omega T$ and $k(\omega)$ is the carrier wave number (frequency). The derivative operators in the above equations are considered as follows:

$$
\begin{aligned}
& \frac{\partial}{\partial T} \rightarrow \frac{\partial}{\partial T}-\epsilon V_{g} \frac{\partial}{\partial \xi}+\epsilon^{2} \frac{\partial}{\partial \tau}, \\
& \frac{\partial}{\partial X} \rightarrow \frac{\partial}{\partial X}+\epsilon \frac{\partial}{\partial \xi} .
\end{aligned}
$$

Now, by substituting Eqs. (15)-(23) into Eqs. (7)-(10), and (14), and collecting power term of $\epsilon$, the first order approximation $(m=1)$ with the first harmonic $(l=1)$ provides the following relation

$$
\begin{aligned}
& i k U_{11}^{(1)}-i \omega N_{11}^{(1)}=0, \\
& i k \lambda N_{11}^{(1)}-i \omega U_{11}^{(1)}-i k \phi_{1}^{(1)}=0, \\
& i k U_{21}^{(2)}-i \omega N_{21}^{(2)}=0, \\
& i k \theta N_{21}^{(1)}+i k \alpha \phi_{1}^{(1)}-i \omega U_{21}^{(1)}=0, \\
& \beta N_{21}^{(1)}-N_{11}^{(1)}-k^{2} \phi_{1}^{(1)}-\gamma_{1} \phi_{1}^{(1)}=0,
\end{aligned}
$$

where $\lambda=3 \sigma_{1}$ and $\theta=3 \sigma_{2}$. Now, these equations detracted to the following pattern

$$
\begin{aligned}
& N_{11}^{(1)}=\frac{k^{2}}{S} \phi_{1}^{(1)}, \\
& U_{11}^{(1)}=\frac{k \omega}{S} \phi_{1}^{(1)}, \\
& N_{21}^{(2)}=\frac{k^{2} \alpha}{A} \phi_{1}^{(1)}, \\
& U_{21}^{(1)}=\frac{k \omega \alpha}{A} \phi_{1}^{(1)},
\end{aligned}
$$

where $A=\omega^{2}-\theta k^{2}$ and $S=\lambda k^{2}-\omega^{2}$. Therefore, the dispersion relation for the DAWs can be written as

$$
\omega^{2}=\frac{k^{2} G \pm k^{2} \sqrt{G^{2}-4 H M}}{2 H},
$$

where $G=\left(\theta k^{2}+\lambda k^{2}+\theta \gamma_{1}+\lambda \gamma_{1}+\alpha \beta+1\right), H=\left(k^{2}+\gamma_{1}\right)$, and $M=\left(\theta \lambda k^{2}+\theta \gamma_{1} \lambda+\theta+\alpha \beta \lambda\right)$. The condition $G^{2}>4 H M$ must be satisfied in order to obtain real and positive values of $\omega$. Normally, two types of DA modes exist, namely, fast $\left(\omega_{f}\right)$ and slow $\left(\omega_{s}\right)$ DA modes according to the positive and negative sign of the Eq. (33). Now, we have studied the dispersion properties by depicting $\omega$ with $k$ in Figs. 1] and 2, which clearly indicates that (a) the value of $\omega_{f}$ increases exponentially with the increasing values of $z_{2}$ for fixed value of $z_{1}, n_{20}$, and $n_{10}$ although, a saturation region begins after a certain value of $k$ (as is clearly shown in Fig. 11); (b) on the other side, the magnitude of $\omega_{s}$ linearly increases with the increase of $z_{2}$ for the fixed value of $z_{1}, n_{20}$, and $n_{10}$ (as is clearly shown in Fig. 2). It is important to mention that in fast DA mode, both positive and negative warm dust species oscillate in phase with electrons and ions, whereas, in slow DA mode, only one of the inertial massive dust components oscillate in phase with electrons and ions, but the other species are in anti-phase with them [17]. Next, with the help of second-order $(m=2$ with $l=1$ ) equations, we obtain the expression of $V_{g}$ like that

$$
\begin{aligned}
V_{g}= & \frac{F_{1}}{2 \omega k\left(A^{2}+\alpha \beta S^{2}\right)}, \\
F_{1}= & \lambda A^{2} k^{2}+\alpha \beta \theta k^{2} S^{2}+\omega^{2} A^{2}+\alpha \beta \omega^{2} S^{2} \\
& -2 A^{2} S^{2}-S A^{2}+\alpha \beta A S^{2} .
\end{aligned}
$$


Now, the second-harmonic mode of the carrier comes from nonlinear self interaction caused by the components $(l=2)$ for the second order $(m=2)$ compressed equations in the following form

$$
\begin{aligned}
& N_{12}^{(2)}=C_{1}\left|\phi_{1}^{(1)}\right|^{2}, \\
& U_{12}^{(2)}=C_{2}\left|\phi_{1}^{(1)}\right|^{2}, \\
& N_{22}^{(2)}=C_{3}\left|\phi_{1}^{(1)}\right|^{2}, \\
& U_{22}^{(2)}=C_{4}\left|\phi_{1}^{(1)}\right|^{2}, \\
& \phi_{2}^{(2)}=C_{5}\left|\phi_{1}^{(1)}\right|^{2},
\end{aligned}
$$

where

$$
\begin{aligned}
C_{1}= & \frac{2 C_{5} k^{2} S^{2}-\left(3 \omega^{2} k^{4}+\lambda k^{6}\right)}{2 S^{3}}, \\
C_{2}= & \frac{\omega C_{1} S^{2}-\omega k^{4}}{k S^{2}}, \\
C_{3}= & \frac{3 \alpha^{2} \omega^{2} k^{4}+\theta \alpha^{2} k^{6}+2 \alpha C_{5} A^{2} k^{2}}{2 A^{3}}, \\
C_{4}= & \frac{\omega C_{3} A^{2}-\omega \alpha^{2} k^{4}}{k A^{2}}, \\
C_{5}= & \frac{F_{2}}{F_{3}}, \\
F_{2}= & 3 \omega^{2} A^{3} k^{4}+\lambda A^{3} k^{6}-2 \gamma_{2} A^{3} S^{3}+3 \beta \alpha^{2} \omega^{2} S^{3} k^{4} \\
& +\beta \theta \alpha^{2} S^{3} k^{6}, \\
F_{3}= & 2 k^{2} S^{2} A^{3}+8 k^{2} A^{3} S^{3}+2 \gamma_{1} A^{3} S^{3}-2 \alpha \beta A^{2} k^{2} S^{3} .
\end{aligned}
$$

After that, we consider the expression for $(m=3, l=0)$ and $(m=2, l=0)$, which leads the zeroth harmonic modes. Finally, we get

$$
\begin{aligned}
& N_{10}^{(2)}=C_{6}\left|\phi_{1}^{(1)}\right|^{2}, \\
& U_{10}^{(2)}=C_{7}\left|\phi_{1}^{(1)}\right|^{2}, \\
& N_{20}^{(2)}=C_{8}\left|\phi_{1}^{(1)}\right|^{2}, \\
& U_{20}^{(2)}=C_{9}\left|\phi_{1}^{(1)}\right|^{2}, \\
& \phi_{0}^{(2)}=C_{10}\left|\phi_{1}^{(1)}\right|^{2},
\end{aligned}
$$

where

$$
\begin{aligned}
C_{6}= & \frac{2 \omega V_{g} k^{3}+\lambda k^{4}+\omega^{2} k^{2}-C_{10} S^{2}}{S^{2}\left(V_{g}^{2}-\lambda\right)}, \\
C_{7}= & \frac{C_{6} V_{g} S^{2}-2 \omega k^{3}}{S^{2}}, \\
C_{8}= & \frac{2 \omega V_{g} \alpha^{2} k^{3}+\theta \alpha^{2} k^{4}+\alpha^{2} \omega^{2} k^{2}+\alpha C_{10} A^{2}}{A^{2}\left(V_{g}^{2}-\theta\right)}, \\
C_{9}= & \frac{C_{8} V_{g} A^{2}-2 \omega \alpha^{2} k^{3}}{A^{2}}, \\
C_{10}= & \frac{F_{4}}{F_{5}}, \\
F_{4}= & \left(A^{2} V_{g}^{2}-\theta A^{2}\right)\left(2 \omega V_{g} k^{3}+\lambda k^{4}+\omega^{2} k^{2}\right) \\
& +\left(V_{g}^{2}-\lambda\right)\left(2 \gamma_{2} A^{2} S^{2} V_{g}^{2}-2 \gamma_{2} \theta A^{2} S^{2}\right) \\
& -\left(2 \omega V_{g} \alpha^{2} k^{3}+\theta \alpha^{2} k^{4}+\alpha^{2} \omega^{2} k^{2}\right) \\
& \left(\beta S^{2} V_{g}^{2}-\beta \lambda S^{2}\right), \\
F_{5}= & \alpha \beta A^{2} S^{2} V_{g}^{2}-\alpha \beta \lambda A^{2} S^{2}+A^{2} S^{2} V_{g}^{2}-\theta A^{2} S^{2} \\
& -\left(V_{g}^{2}-\lambda\right)\left(\gamma_{1} A^{2} S^{2} V_{g}^{2}-\gamma_{1} \theta A^{2} S^{2}\right) .
\end{aligned}
$$

Later, with the help of Eqs. (29) - (44), the third harmonic $(m=3)$ with $(l=1)$ modes can be accomplished. Thus, we can write the NLS equation as;

$$
i \frac{\partial \Phi}{\partial \tau}+P \frac{\partial^{2} \Phi}{\partial \xi^{2}}+Q|\Phi|^{2} \Phi=0
$$

where $\Phi=\phi_{1}^{(1)}$ for simplicity and $P(Q)$ is the dispersion (nonlinear) coefficient, and is written by

$$
P=\frac{F_{6}}{2 \omega A S k^{2}\left(A^{2}+\alpha \beta S^{2}\right)},
$$

and

$$
Q=\frac{F_{7}}{2 \omega k^{2}\left(A^{2}+\alpha \beta S^{2}\right)},
$$

where

$$
\begin{aligned}
F_{6}= & \left(\omega V_{g} A^{3}-\lambda k A^{3}\right)\left(\lambda k^{3}-2 \omega V_{g} k^{2}+k \omega^{2}-k S\right) \\
& +\left(k V_{g} A^{3}-\omega A^{3}\right)\left(\lambda \omega k^{2}-2 k V_{g} \omega^{2}+\omega^{3}-k S V_{g}\right) \\
& -\left(\theta k^{3}-2 \omega V_{g} k^{2}+k \omega^{2}+A k\right)\left(\alpha \beta \omega V_{g} S^{3}\right. \\
& \left.-\alpha \beta \theta k S^{3}\right)-A^{3} S^{3}-\left(\alpha \beta k V_{g} S^{3}-\alpha \beta \omega S^{3}\right) \\
& \left(\theta \omega k^{2}-2 k V_{g} \omega^{2}+\omega^{3}+A k V_{g}\right), \\
F_{7}= & 2 \gamma_{2} C_{5} A^{2} S^{2}+2 \gamma_{2} C_{10} A^{2} S^{2}+3 \gamma_{3} A^{2} S^{2} \\
& -2 \omega C_{2} A^{2} k^{3}-2 \omega C_{7} A^{2} k^{3}-2 \alpha \beta \omega C_{4} S^{2} k^{3} \\
- & 2 \alpha \beta \omega C_{9} S^{2} k^{3}-C_{1} \omega^{2} A^{2} k^{2}-C_{6} \omega^{2} A^{2} k^{2} \\
- & \lambda C_{1} A^{2} k^{4}-\lambda C_{6} A^{2} k^{4}-\alpha \beta C_{3} \omega^{2} k^{2} S^{2} \\
- & \alpha \beta C_{8} \omega^{2} k^{2} S^{2}-\alpha \beta \theta C_{3} S^{2} k^{4}-\alpha \beta \theta C_{8} S^{2} k^{4} .
\end{aligned}
$$




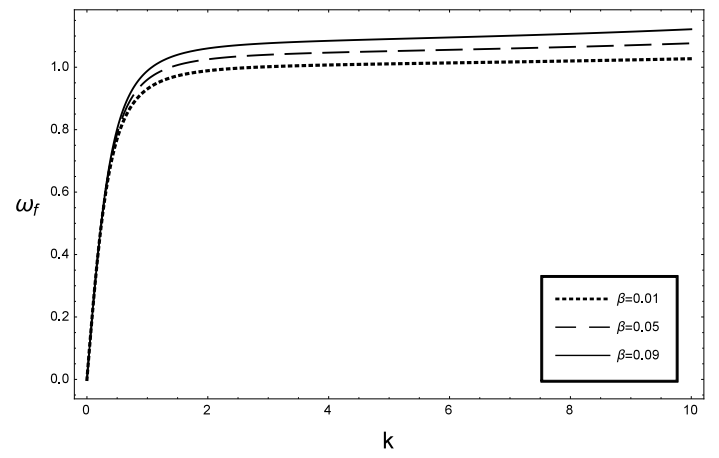

FIG. 1: The variation of $\omega_{f}$ with $k$ for different values of $\beta$; along with $\alpha=2.0, \delta=0.3, \mu_{i}=0.4, \sigma_{1}=0.0001$, $\sigma_{2}=0.001$, and $q=1.5$.

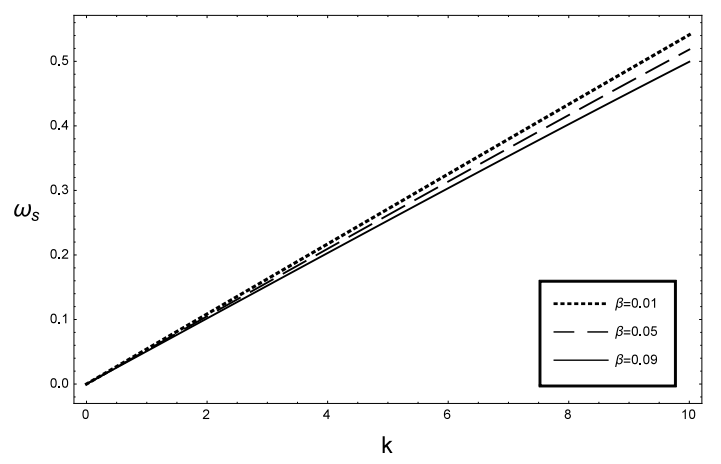

FIG. 2: The variation of $\omega_{s}$ with $k$ for different values of $\beta$; along with $\alpha=2.0, \delta=0.3, \mu_{i}=0.4, \sigma_{1}=0.0001$, $\sigma_{2}=0.001$, and $q=1.5$.

\section{STABILITY OF DAWS}

To study the MI of DAWs, we consider the linear solution of the NLS equation (45) in the form $\Phi=$ $\hat{\Phi} e^{i Q|\hat{\Phi}|^{2} \tau}+$ c.c (c.c denotes the complex conjugate), where $\hat{\Phi}=\hat{\Phi}_{0}+\epsilon \hat{\Phi}_{1}$ and $\hat{\Phi}_{1}=\hat{\Phi}_{1,0} e^{i(\tilde{k} \xi-\tilde{\omega} \tau)}+$ c.c (the perturbed wave number $\tilde{k}$ and the frequency $\tilde{\omega}$ are different from $k$ and $\omega$ ). Now, by substituting these values in Eq. (45) the following nonlinear dispersion relation can be obtained as 3033 . 33

$$
\tilde{\omega}^{2}=P^{2} \tilde{k}^{2}\left(\tilde{k}^{2}-\frac{2\left|\hat{\Phi}_{0}\right|^{2}}{P / Q}\right) .
$$

It is clear from Eq. (46) that the DAWs will be modulationally stable (unstable) in the range of values of $\tilde{k}$ in which $P / Q$ is negative (positive). When $P / Q \rightarrow \pm \infty$, the corresponding value of $k\left(=k_{c}\right)$ is called the critical or threshold wave number $\left(k_{c}\right)$ for the onset of MI. The variation of $P / Q$ with $k$ for $\mu_{i}$ and $\alpha$ are shown in Figs. 3 and 4, respectively, which clearly indicate that (a) the value of $k_{c}$ increases with the increase of $n_{i 0}$ for fixed value of $z_{1}$ and $n_{10}$; (b) on the other hand, $k_{c}$ value

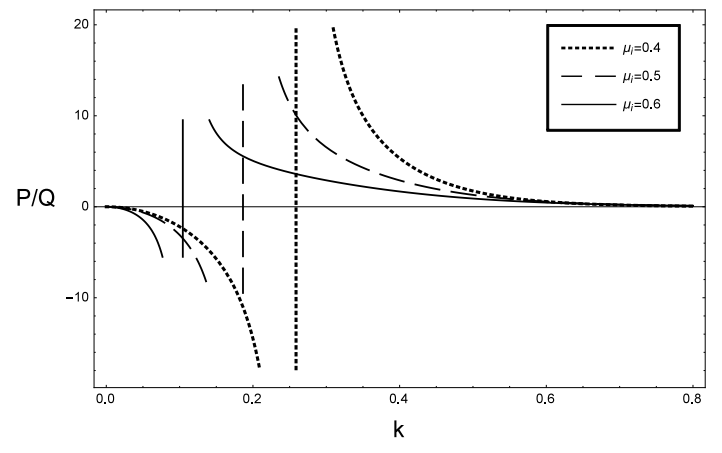

FIG. 3: The variation of $P / Q$ with $k$ for different values of $\mu_{i}$; along with $\alpha=2.0, \beta=0.07, \delta=0.3, \sigma_{1}=0.0001$, $\sigma_{2}=0.001, \omega_{f}$, and $q=1.5$.

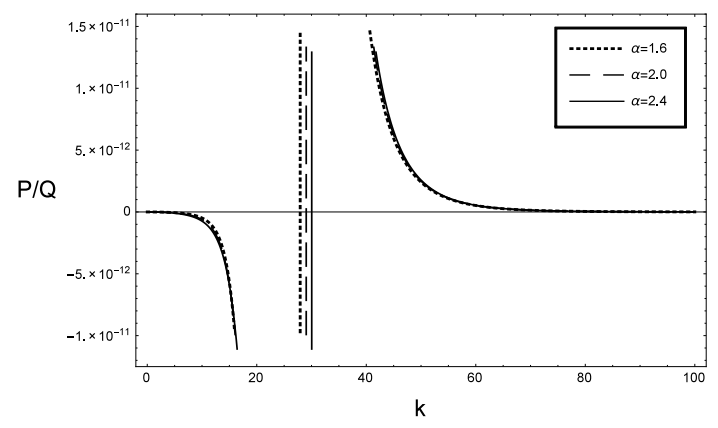

FIG. 4: The variation of $P / Q$ with $k$ for different values of $\alpha$; along with $\beta=0.07, \delta=0.3, \mu_{i}=0.4, \sigma_{1}=0.0001$, $\sigma_{2}=0.001, \omega_{s}$, and $q=1.5$.

decreases with the increase of $m_{2}$ for fixed value of $m_{1}$, $z_{2}$, and $z_{1}$. It also can be written from Eq. (46) that the growth rate $(\Gamma)$ of the modulationally unstable region for the DAWs [when $P / Q>0$ and $\tilde{k}<\tilde{k}_{c}=\left(2 Q\left|\hat{\Phi}_{0}\right|^{2} / P\right)^{1 / 2}$ ] as

$$
\Gamma=|P| \tilde{k}^{2}\left(\frac{\tilde{k}_{c}^{2}}{\tilde{k}^{2}}-1\right)^{1 / 2} .
$$

Moreover, we have graphically shown how the $\Gamma$ varies with $\tilde{k}$ of different values of $\alpha$ and $q$ in Figs. $5-9$.

It is obvious from Figs. [5-77 that (a) the maximum value of $\Gamma$ increases with the increase of the values of $z_{2}$ for fixed value of $q=1$ (Maxwellian limit), $z_{1}, m_{1}$, and $m_{2}$ (as is clearly shown in Fig. 5); (b) the magnitude of $\Gamma$ also increases with the increase of $z_{2}$ for fixed value of $q=1.5$ (subextensive range), $z_{1}, m_{1}$, and $m_{2}$ (as is clearly shown in Fig. 6); (c) Moreover, $\Gamma$ increases with the increase of $z_{2}$ for fixed value of $q=-0.6$ (superextensive range), $z_{1}, m_{1}$, and $m_{2}$ (as clearly shown in Fig. 77). The physics of this result is that, since the nonlinearity increases with the increase of the values of $\alpha$, the growth rate of DAWs increase.

It can be observed from Figs. 8 and 9 that the maximum value of $\Gamma$ increases (decreases) with the decrease 


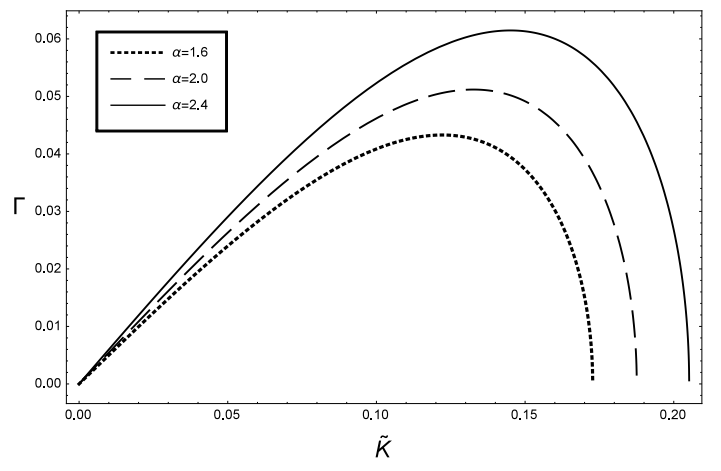

FIG. 5: The variation of MI growth rate $(\Gamma)$ with $\tilde{k}$ for different values of $\alpha$; along with $\beta=0.07, \delta=0.3, \mu_{i}=0.4$, $\sigma_{1}=0.0001, \sigma_{2}=0.001, \omega_{f}, q=1.0, \Phi_{0}=0.5$, and $k=0.3$.

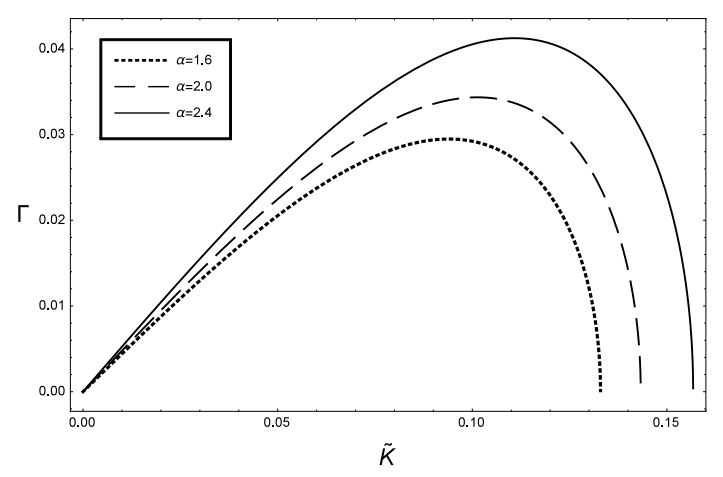

FIG. 6: The variation of MI growth rate $(\Gamma)$ with $\tilde{k}$ for different values of $\alpha$; along with $\beta=0.07, \delta=0.3, \mu_{i}=0.4$, $\sigma_{1}=0.0001, \sigma_{2}=0.001, \omega_{f}, q=1.5, \Phi_{0}=0.5$, and $k=0.3$.

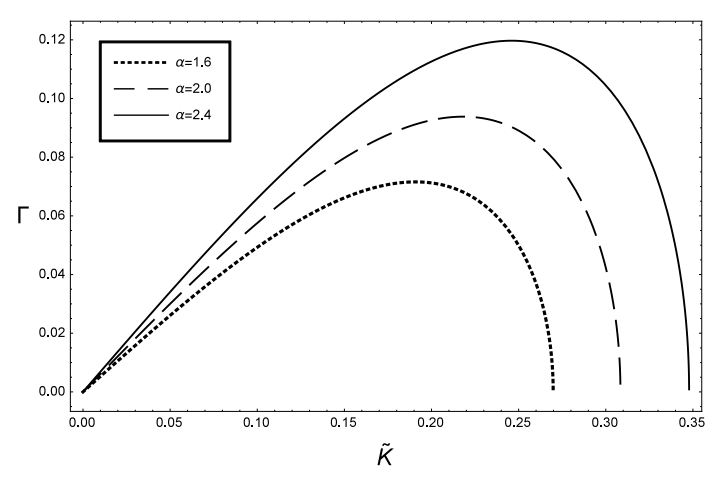

FIG. 7: The variation of MI growth rate $(\Gamma)$ with $\tilde{k}$ for different values of $\alpha$; along with $\beta=0.07, \delta=0.3, \mu_{i}=0.4$, $\sigma_{1}=0.0001, \sigma_{2}=0.001, \omega_{f}, q=-0.6, \Phi_{0}=0.5$, and $k=0.3$.

(increase) of the values of $q>1(q<1)$ (as is clearly shown in Fig. 8 and 9). It can be concluded here that the variation of $\Gamma$ with respect to $\tilde{k}$ is independent on the sign of the $q$.

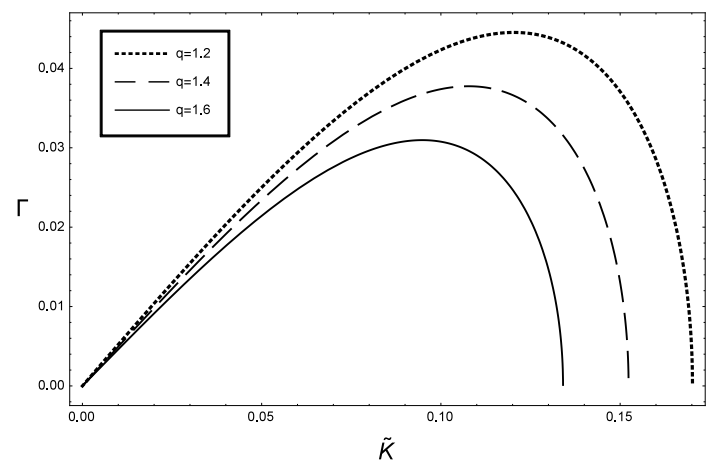

FIG. 8: The variation of MI growth rate $(\Gamma)$ with $\tilde{k}$ for $q$ (within sub-extensive range); along with $\beta=0.07, \delta=0.3$, $\mu_{i}=0.4, \sigma_{1}=0.0001, \sigma_{2}=0.001, \omega_{f}, \Phi_{0}=0.5$, and $k=0.3$.

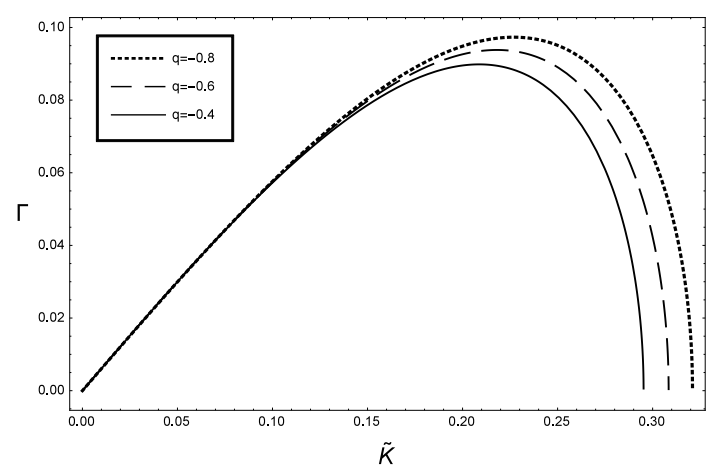

FIG. 9: The variation of MI growth rate $(\Gamma)$ with $\tilde{k}$ for $q$ (within super-extensive range); along with $\beta=0.07, \delta=0.3$, $\mu_{i}=0.4, \sigma_{1}=0.0001, \sigma_{2}=0.001, \omega_{f}, \Phi_{0}=0.5$, and $k=0.3$.

\section{ENVELOPE SOLITONS}

There are two types of envelope solitonic solutions exist, namely, bright and dark envelope solitons, depending on the sign of the coefficients $P$ and $Q$.

\section{A. Bright envelope solitons}

When $P / Q>0$, the expression of the bright envelope solitonic solution of Eq. (45) can be written as 30 33.

$$
\begin{aligned}
\Phi(\xi, \tau)= & {\left[\psi_{0} \operatorname{sech}^{2}\left(\frac{\xi-U \tau}{W}\right)\right]^{1 / 2} } \\
& \times \exp \left[\frac{i}{2 P}\left\{U \xi+\left(\Omega_{0}-\frac{U^{2}}{2}\right) \tau\right\}\right],
\end{aligned}
$$

where $\psi_{0}$ indicates the envelope amplitude, $U$ is the travelling speed of the localized pulse, $W$ is the pulse width, which can be written as $W=\left(2 P \psi_{0} / Q\right)^{1 / 2}$, and $\Omega_{0}$ is the oscillating frequency for $U=0$. The bright envelope soliton is depicted in Fig. 10. The amplitude of the bright envelope solitons remains constant but the width 


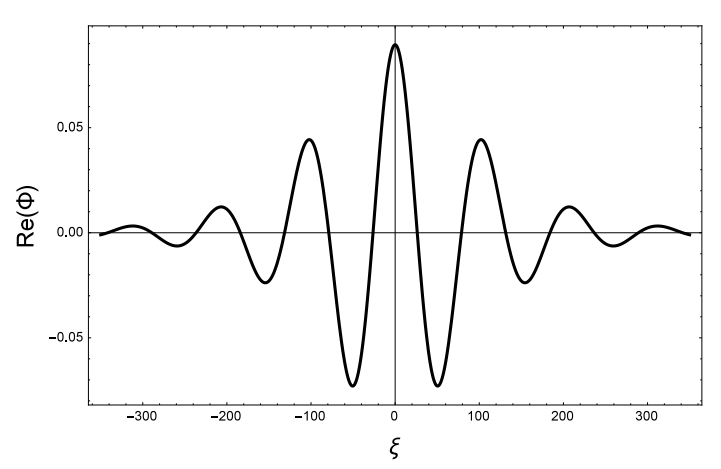

FIG. 10: The variation of $\operatorname{Re}(\Phi)$ with $\xi$ for bright envelope solitons; along with $\alpha=2.0, \beta=0.07, \delta=0.3, \mu_{i}=0.4$, $\sigma_{1}=0.0001, \sigma_{2}=0.001, \tau=0, \psi_{0}=0.008, \omega_{f}, q=1.5$, $k=0.3, \Omega_{0}=0.4$, and $U=0.4$.

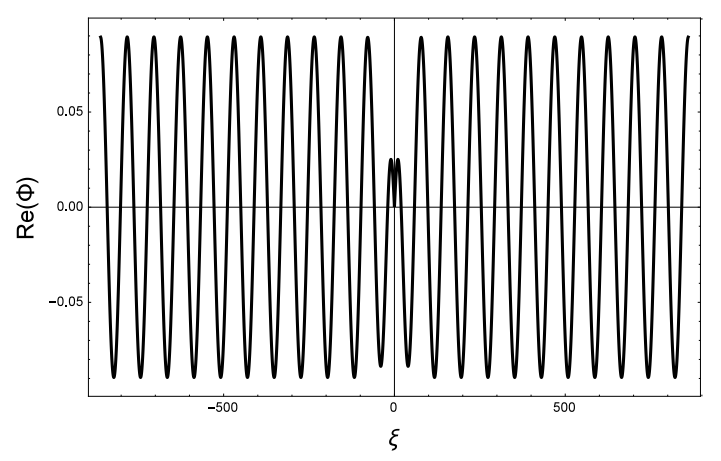

FIG. 11: The variation of $\operatorname{Re}(\Phi)$ with $\xi$ for dark envelope solitons; along with $\alpha=2.0, \beta=0.07, \delta=0.3, \mu_{i}=0.4$, $\sigma_{1}=0.0001, \sigma_{2}=0.001, \tau=0, \psi_{0}=0.008, \omega_{f}, q=1.5$, $k=0.1, \Omega_{0}=0.4$, and $U=0.4$.

of the bright envelope solitons increases as we increase the value of the $q$-distributed electron temperature $T_{e}$, for fixed value of ion temperature $T_{i}$ (via $\delta$ ).

\section{B. Dark envelope solitons}

As we know before that the condition for dark envelope soliton is $P / Q<0$. So, the dark envelope soliton solution of Eq. (45) can be written as 30 33]

$$
\begin{aligned}
\Phi(\xi, \tau)= & {\left[\psi_{0} \tanh ^{2}\left(\frac{\xi-U \tau}{W}\right)\right]^{1 / 2} } \\
& \times \exp \left[\frac{i}{2 P}\left\{U \xi-\left(\frac{U^{2}}{2}-2 P Q \psi_{0}\right) \tau\right\}\right] .
\end{aligned}
$$

The dark envelope soliton, obtained from Eq. (49) is shown in Fig. 11]

\section{DISCUSSION}

We have studied an unmagnetized realistic space dusty plasma system consists of non-extensive $q$-distributed electrons, isothermal ions, positively charged warm dust particles as well as negatively charged warm dust particles. The reductive perturbation method is used to derive the NLS equation. The results that have been found from our investigation can be summarized as follows

1. The fast DA mode increases exponentially with the increasing values of $z_{2}$ for fixed value of $z_{1}, n_{20}$ (via $\beta$ ), and $n_{10}$. On the other hand, the slow DA mode linearly increases with the increase of $z_{2}$ for the fixed value of $z_{1}, n_{20}$, and $n_{10}(\operatorname{via} \beta)$.

2. The DAWs is modulationally stable (unstable) in the range of values of $k$ in which the ratio $P / Q$ is $P / Q<0(P / Q>0)$.

3. The value of $k_{c}$ increases with the increase of $n_{i 0}$ for fixed value of $z_{1}$ and $n_{10}\left(\right.$ via $\left.\mu_{i}\right)$. On the other hand, $k_{c}$ value decreases with the increase of $m_{2}$ for fixed value of $m_{1}, z_{2}$, and $z_{1}$ (via $\alpha$ ).

4. The value of $\Gamma$ increases with the decrease of $z_{2}$ for fixed value of $z_{1}, m_{1}, m_{2}$, and $q$ (where the values of $q$ lies in the range of $q>1, q=1$, and $q<1$, respectively).

5. The maximum value of $\Gamma$ increases (decreases) with the decrease (increase) of the values of $q>1(q<$ $1)$. So, the growth rate is independent on the sign of the $q$.

6. The amplitude of the bright envelope solitons remains constant but the width of the bright envelope solitons increases as we increase the value of the $q$-distributed electron temperature $T_{e}$, for fixed value of ion temperature $T_{i}$ (via $\delta$ ).

The results of our present investigation will be useful in understanding the nonlinear phenomena both in space (e.g. Jupiters magnetosphere 11, 13, 16, 17], upper mesosphere [14], comets tails [12, 13], etc.) and laboratory (viz. direct current and radio-frequency discharges, plasma processing reactors, fusion plasma devices [1], solid-fuel combustion products [1], etc.) plasma system containing nonextensive $q$-distributed electrons, isothermal ions, negatively and positively charged warm dust.

[4] E. C. Whipple, T. G. Northrop, and D. A. Mendis, J. Geophys. Res. 90, 7405 (1985).

[5] A. Mamun, R. A. Cairns, and P. K. Shukla, Phys. Plas-

mas 3, 702 (1996).
[1] P. K. Shukla and A. A. Mamun, Introdustion to Dusty Plasma Physics (Institute of Physics, Bristol, 2002).

[2] F. Verheest, Space Sci. Rev. 77, 267 (1996).

[3] C. K. Goertz, Rev. Geophys. 27, 271 (1989). 
[6] N. N. Rao, P. K. Shukla, and M. Y. Yu, Planet. Space. Sci. 38, 543 (1990).

[7] N. D’ Angelo, Planet. Space. Sci. 38, 1143 (1990).

[8] A. A. Mamun and R. Schlickeiser, Phys. Plasmas 23, 034502 (2016).

[9] V. W. Chow, D. A. Mendis, and M. Rosenberg, J. Geophys. Res. 98, 19065 (1993).

[10] D. A. Mendis and M. Rosenberg, Annu. Rev. Astron. Astrophys. 32, 419 (1994).

[11] M. Horányi, G. E. Morfill, and E. Grün, Nature (London) 363, 144 (1993).

[12] D. A. Mendis and M. Hornyi, in Cometary Plasma Processes, Monograph 61 (American Geophysical Union, Washington, DC, 1991).

[13] M. Horányi, Annu. Rev. Astron. Astrophys. 34, 3831996.

[14] O. Havnes, J. Trim, T. Blix et al., J. Geophys. Res. 101, 10839 (1996).

[15] V. E. Fortov et al., J. Exp. Theor. Phys. 87, 1087 (1998)

[16] A. A. Mamun, Phys. Rev. E 77, 026406 (2008).

[17] N. A. Chowdhury, A. Mannan, and A. A. Mamun, Phys. Plasmas 24, 113701 (2017).

[18] T. Akhter, M. M. Hossain, and A. A. Mamun, Phys. Plasmas 19, 093707 (2012).

[19] A. Renyi, Acta Math. Acad. Sci. Hung. 6, 285 (1955).
[20] C. Tsallis, J. Stat. Phys. 52, 479 (1988).

[21] I. Driouch and H. Chatei, Eur. Phys. J. D 9, 71 (2017).

[22] A. E. Dubinov, Plasma Phys. Rep. 35, 991 (2009).

[23] T. S. Gill, A. S. Bains, and C. Bedi, Phys. Plasmas 17, 013701 (2010).

[24] I. Tasnim, M. M. Masud, M. Asaduzzaman, and A. A. Mamun, Chaos 23, 013147 (2013).

[25] N. A. Chowdhury, M. M. Hasan, A. Mannan, and A. A. Mamun, Vacuum 147, 31-37 (2018).

[26] A.P. Misra and A. R. Chowdhury, Eur. Phys. J. D 39, 4957 (2006).

[27] F. Sayed and A. A. Mamun, Phys. Plasmas 14, 014501 (2007).

[28] W. F. El-Taibany, Phys. Plasmas 20, 093701 (2013).

[29] N. A. Chowdhury, A. Mannan, M. M. Hasan, and A. A. Mamun, Chaos 27, 093105 (2017).

[30] S. Sultana and I. Kourakis, Plasma Phys. Control. Fusion 53, 045003 (2011).

[31] I. Kourakis and P.K. Sukla, Nonlinear Proc. Geophys. 12, 407 (2005).

[32] R. Fedele and H. Schamel, Eur. Phys. J. B 27, 313 (2002).

[33] R. Fedele, Phys. Scr. 65, 502 (2002). 